Published in final edited form as:

Am J Kidney Dis. 2019 January ; 73(1): 21-30. doi:10.1053/j.ajkd.2018.07.015.

\title{
Effects of Intensive Blood Pressure Lowering on Kidney Tubule Injury in CKD: A Longitudinal Subgroup Analysis in SPRINT
}

\author{
Rakesh Malhotra, \\ Division of Nephrology and Hypertension, Department of Medicine, University of California San \\ Diego, San Diego \\ Imperial Valley Family Care Medical Group, El Centro, CA \\ Timothy Craven, \\ Department of Biostatistical Sciences, Wake Forest School of Medicine, Winston-Salem, NC \\ Walter T. Ambrosius, \\ Department of Biostatistical Sciences, Wake Forest School of Medicine, Winston-Salem, NC \\ Anthony A. Killeen, \\ Department of Laboratory Medicine and Pathology, University of Minnesota, Minneapolis, MN \\ William E. Haley, \\ Division of Nephrology, Department of Medicine, Mayo Clinic, Jacksonville, FL
}

\author{
Alfred K. Cheung, \\ Division of Nephrology \& Hypertension, Department of Internal Medicine, University of Utah; \\ Medical Service, Veterans Affairs Salt Lake City Healthcare System, Salt Lake City, UT \\ Michel Chonchol, \\ Division of Nephrology \& Hypertension, Department of Medicine, University of Colorado, Denver, \\ $\mathrm{CO}$

\section{Mark Sarnak,} \\ Division of Nephrology, Department of Medicine, Tufts Medical Center, Boston, MA \\ Chirag R. Parikh, \\ Division of Nephrology, Department of Medicine, Yale University School of Medicine, New Haven, \\ CT
}

\footnotetext{
Address for Correspondence: Joachim H. Ix, MD, MAS, Division of Nephrology-Hypertension, 3350 La Jolla Village Dr, Mail Code 9111-H, San Diego, CA 92161. joeix@ucsd.edu.

Authors' Contributions: Study concept and design: MGS, JHI; acquisition, analysis, or interpretation of data: RM, TC, WA, AAK, WEH, AKC, MC, MS, CRP, MGS, JHI; statistical analysis: TC, WA; obtained funding: MGS, JHI. Each author contributed important intellectual content during manuscript drafting or revision and accepts accountability for the overall work by ensuring that questions pertaining to the accuracy or integrity of any portion of the work are appropriately investigated and resolved.

Disclaimer: The content is solely the responsibility of the authors and does not necessarily represent the official views of the NIH, the US Department of Veterans Affairs, or the US Government.

Prior Presentation: An abstract based on these findings was presented at the American Society of Nephrology's Kidney Week 2017 meeting in New Orleans, LA, October 31 to November 5, 2017.

Correction Notice: This article was amended on November 14, 2018 to correct the Financial Disclosure statement. SPRINT Research Group Members: A list of the members of the SPRINT Research Group appears in the supplementary material accompanying Ambrosius et al ${ }^{18}$ and Wright et al. 13
} 


\author{
Michael G. Shlipak ${ }^{\#}$, \\ Division of General Internal Medicine, San Francisco VA Medical Center \\ Kidney Health Research Collaborative, San Francisco VA Medical Center and University of \\ California, San Francisco \\ Joachim H. IX \\ Division of Nephrology and Hypertension, Department of Medicine, University of California San \\ Diego, San Diego \\ Division of Preventive Medicine, Department of Family Medicine and Public Health, University of \\ California San Diego, San Diego \\ Nephrology Section, Veterans Affairs San Diego Healthcare System, La Jolla, CA

\section{SPRINT Research Group} \\ \# These authors contributed equally to this work.
}

\title{
Abstract
}

Background-Random assignment to the intensive systolic blood pressure (SBP) arm $(<120$ $\mathrm{mm} \mathrm{Hg}$ ) in the Systolic Blood Pressure Intervention Trial (SPRINT) resulted in more rapid declines in estimated glomerular filtration rates (eGFRs) than in the standard arm (SBP $<140 \mathrm{~mm}$ $\mathrm{Hg}$ ). Whether this change reflects hemodynamic effects or accelerated intrinsic kidney damage is unknown.

Study Design—Longitudinal subgroup analysis of clinical trial participants.

Settings \& Participants-Random sample of SPRINT participants with prevalent chronic kidney disease (CKD) defined as eGFR $<60 \mathrm{~mL} / \mathrm{min} / 1.73 \mathrm{~m}^{2}$ by the CKD-EPI (CKD Epidemiology Collaboration) creatinine-cystatin $\mathrm{C}$ equation at baseline.

Outcomes \& Measurements-Urine biomarkers of tubule function $\left(\beta_{2}\right.$-microglobulin [B2M], $\mathrm{a}_{1}$-microglobulin $[\mathrm{A} 1 \mathrm{M}]$ ), and uromodulin), injury (interleukin 18 , kidney injury molecule 1 , and neutrophil gelatinase-associated lipocalin), inflammation (monocyte chemoattractant protein 1), and repair (human cartilage glycoprotein 40) at baseline, year 1, and year 4. Biomarkers were indexed to urine creatinine concentration and changes between arms were evaluated using mixedeffects linear models and an intention-to-treat approach.

Results—978 SPRINT participants (519 in the intensive and 459 in the standard arm) with prevalent CKD were included. Mean age was $72 \pm 9$ years and eGFR was $46.1 \pm 9.4 \mathrm{~mL} / \mathrm{min} / 1.73$ $\mathrm{m}^{2}$ at baseline. Clinical characteristics, eGFR, urinary albumin-creatinine ratio, and all 8 biomarker values were similar across arms at baseline. Compared to the standard arm, eGFR was lower by 2.9 and $3.3 \mathrm{~mL} / \mathrm{min} / 1.73 \mathrm{~m}^{2}$ in the intensive arm at year 1 and year 4 . None of the 8 tubule marker levels was higher in the intensive arm compared to the standard arm at year 1 or year 4. Two tubule function markers (B2M and A1M) were 29\% (95\% CI, 10\%-43\%) and 24\% $(95 \% \mathrm{CI}, 10 \%-36 \%)$ lower at year 1 in the intensive versus standard arm, respectively.

Limitations-Exclusion of persons with diabetes, and few participants had advanced CKD. Conclusions-Among participants with CKD in SPRINT, random assignment to the intensive SBP arm did not increase any levels of 8 urine biomarkers of tubule cell damage despite loss of 
eGFR. These findings support the hypothesis that eGFR declines in the intensive arm of SPRINT predominantly reflect hemodynamic changes rather than intrinsic damage to kidney tubule cells.

Hypertension is common and a significant risk factor for cardiovascular disease (CVD) ${ }^{1-3}$

A number of clinical trials and meta-analyses have demonstrated that treatment of hypertension lowers risk for CVD and all-cause mortality. ${ }^{4-6}$ However, the effects of blood pressure (BP) lowering on chronic kidney disease (CKD) progression are less clear ${ }^{7-10}$ because treating to lower BP targets results in higher risk for acute kidney injury (AKI) and more rapid loss of estimated glomerular filtration rate (eGFR). ${ }^{8,11,12}$ The risks for both AKI and eGFR loss may be particularly concerning in patients with prevalent CKD because they have lower eGFRs at baseline and therefore may be least able to tolerate additional kidney insults. Balancing the risks and benefits, the appropriate BP target in patients with CKD remains an area of controversy.

Recently, SPRINT (Systolic Blood Pressure Intervention Trial) compared the effects of intensive BP lowering (systolic BP [SBP] target of $<120 \mathrm{~mm} \mathrm{Hg}$ ) to standard BP control (SBP $<140 \mathrm{~mm} \mathrm{Hg}$ ) on risk for CVD events. ${ }^{13}$ SPRINT enrolled hypertensive individuals without diabetes or prior stroke, but with high CVD risk. Approximately $30 \%(n=2,646)$ had CKD at baseline. SPRINT was terminated early at the recommendation of the data safety monitoring board due to substantial benefit for the primary CVD end point and lower mortality risk in patients randomly assigned to the intensive BP arm. Comparing subgroups with and without CKD at baseline, there was no evidence of heterogeneity for the CVD end point, and yet the intensive arm experienced more rapid loss of eGFR and higher risk for AKI. ${ }^{13,14}$ The effect of intensive BP lowering on eGFR was most pronounced during the first 6 months of treatment, which has led to speculation that the change may represent hemodynamic effects of more intensive BP lowering on eGFR rather than intrinsic kidney damage. ${ }^{14}$

During the past decade, several urinary biomarkers of kidney tubule function and injury have been identified. ${ }^{15}$ Although evaluated initially as diagnostic tests for AKI, subsequent studies have demonstrated that higher urine concentrations of these markers also predict more rapid loss of kidney function in community-living individuals without AKI. ${ }^{16,17}$ Because abnormal levels of these biomarkers would suggest intrinsic kidney tubule cell injury and/or dysfunction, they provide an opportunity to assess the influence of intensive BP lowering on kidney health above and beyond eGFR loss. Therefore, we measured urinary biomarkers that reflect kidney tubule function, inflammation, injury, and repair in a subset of SPRINT participants with CKD at baseline. A priori, we hypothesized that the predominant cause for the greater change in eGFR in the intensive arm of SPRINT reflected hemodynamic changes and therefore examined changes in markers of kidney tubule health by treatment arm assignment.

\section{Methods}

Study Design

The trial design ${ }^{18}$ and results for the primary CVD end point ${ }^{13}$ of SPRINT have been reported previously. Briefly, SPRINT is an open-label clinical trial that randomly assigned 
persons with SBP $\geq 130 \mathrm{~mm} \mathrm{Hg}$ and high risk for CVD events to an SBP target of $<120 \mathrm{~mm}$ $\mathrm{Hg}$ ("intensive") versus <140 mm Hg ("standard"). ${ }^{18}$ Participants were recruited from 102 centers in the United States and Puerto Rico. Inclusion criteria required age of 50 years and older, SBP of 130 to $180 \mathrm{~mm} \mathrm{Hg}$, and increased risk for CVD events (prior clinical or subclinical CVD other than stroke, 10-year risk for CVD $\geq 15 \%$ based on the Framingham risk score, CKD defined as eGFR of $20-59 \mathrm{~mL} / \mathrm{min} / 1.73 \mathrm{~m}^{2}$, or age $\geq 75$ years). Major exclusion criteria included diabetes mellitus, proteinuria with protein excretion $>1 \mathrm{~g} / \mathrm{d}$, polycystic kidney disease, prior stroke or transient ischemic attack, symptomatic heart failure, or left ventricular ejection fraction $<35 \%$. A total of 9,361 participants were enrolled between November 2010 and March 2013. All participants provided written informed consent. Institutional review boards of all participating institutions approved the study.

We measured serum cystatin $\mathrm{C}$ in all SPRINT participants and defined the subset with eGFRs $<60 \mathrm{~mL} / \mathrm{min} / 1.73 \mathrm{~m}^{2}$ using the CKD-EPI (CKD Epidemiology Collaboration) creatinine-cystatin C equation. From the 2,646 participants with baseline eGFRs < 60 $\mathrm{mL} / \mathrm{min} / 1.73 \mathrm{~m}^{2}, 1,000$ individuals were selected using simple random sampling for participation in this study. Twenty-two individuals had unavailable urine specimens and were excluded from further analysis. Thus, the final analytic sample for this study included 978 participants with CKD at baseline.

In SPRINT, participants were randomly assigned in a 1:1 ratio to the intensive or standard arm. Antihypertensive regimens were adjusted to maintain SBP according to the randomly assigned BP study groups. ${ }^{18}$ Participants attended visits monthly for the first 3 months and then every 3 months thereafter, and clinical and laboratory data were obtained at these visits. Venous blood and urine specimens were immediately processed, shipped overnight on ice packs, and stored at $-80^{\circ} \mathrm{C}$ at the central laboratory for use in future studies. We have previously reported eGFR decline and changes in urinary albumin-creatinine ratio (ACR) in the CKD subgroup ${ }^{14}$ and showed that random assignment to the intensive group was associated with more rapid decline in eGFR and a decrease in urinary ACR relative to the standard group among participants with CKD.

\section{Kidney Tubule Biomarker Measurements}

Urinary specimens from the baseline, 12-month, and 48-month SPRINT visits were stored at $-80^{\circ} \mathrm{C}$ until thawing for kidney tubule cell damage biomarker measurements. All specimens were thawed at once and measurements were completed en bloc to minimize any influence of analytic drift on longitudinal changes in biomarkers. We chose 8 distinct urinary markers because they reflect aspects of kidney tubule biology, including tubule function $\left(\beta_{2^{-}}\right.$ microglobulin $[\mathrm{B} 2 \mathrm{M}], \mathrm{a}_{1}$-microglobulin [A1M], and uromodulin [UMOD]), injury (interleukin 18 [IL-18], kidney injury molecule 1 [KIM-1], and neutrophil gelatinaseassociated lipocalin [NGAL]), inflammation (monocyte chemoattractant protein 1 [MCP-1]), and repair (human cartilage glycoprotein 39 [YKL-40]) and are known to be associated with CKD progression. ${ }^{19-24}$

B2M and A1M are low-molecular-weight proteins that are freely filtered at the glomerulus and then reabsorbed by the proximal tubule. We have previously demonstrated that higher 
levels of these proteins in urine are associated with kidney function decline among persons infected with human immunodeficiency virus (HIV). ${ }^{19}$ UMOD is a $95-\mathrm{kDa}$ glycoprotein synthesized exclusively by kidney tubules. Studies have shown that higher UMOD levels are associated with kidney size and eGFR, and lower UMOD levels are independently associated with CKD progression. ${ }^{20}$ IL-18, KIM-1, and NGAL have been studied extensively as markers of tubular injury, with urine levels increasing by several-fold in response to ischemic or inflammatory kidney injury. ${ }^{21,22} \mathrm{MCP}-1$ is a chemokine that attracts macrophages to the site of injury, and we have shown strong associations of this marker with CKD progression in kidney transplant recipients. ${ }^{23}$ YKL-40 functions as a mediator of the reparative response to tubular injury. ${ }^{24}$ Taken together, the selected urinary markers measure the interlinked axes of inflammation, tubular injury and atrophy, and tubulointerstitial fibrosis, which are hallmarks of progressive CKD.

Urinary biomarkers were measured centrally at the Laboratory for Clinical Biochemistry Research at the University of Vermont. Most urinary biomarkers (all except A1M) were measured using multiplex assays on a MESO Scale Diagnostics (MSD) platform. Urinary YKL-40, IL-18, MCP-1, and KIM-1 measures were conducted together on a 4-plex assay. Analytic ranges were 10 to $500,000 \mathrm{ng} / \mathrm{mL}, 2$ to $10,000 \mathrm{ng} / \mathrm{mL}, 3$ to $10,000 \mathrm{pg} / \mathrm{mL}$, and 4 to $200,000 \mathrm{pg} / \mathrm{mL}$ for YKL-40, IL-18, MCP-1, and KIM-1, respectively. Interassay coefficients of variation (CVs) ranged across the analytic range from $6.5 \%$ to $11.1 \%, 4.9 \%$ to $13.7 \%$, $7.1 \%$ to $12.0 \%$, and $6.1 \%$ to $13.0 \%$, respectively. A second 3-plex MSD assay was used to measure urinary B2M, UMOD, and NGAL; for these, analytic ranges were 1.2 to 5,020 $\mathrm{ng} / \mathrm{mL}, 0.6$ to $2,510 \mathrm{ng} / \mathrm{mL}$, and 6 to $251,000 \mathrm{ng} / \mathrm{mL}$, respectively, and interassay CVs were $15 \%$ to $16 \%, 13 \%$ to $16 \%$, and $11 \%$ to $19 \%$. For urinary A1M, we used a Siemens nephelometric assay with a detectable range from 5 to $480 \mathrm{mg} / \mathrm{L}$ and interassay CVs ranging from $3.5 \%$ to $8.8 \%$ across the analytic range. Each marker was measured twice in each urine specimen and results were averaged to improve precision. Urine creatinine was measured using an enzymatic procedure (Roche), and urine albumin, using a nephelometric method (Siemens). ${ }^{14}$

\section{Statistical Analysis}

Descriptive statistics were computed and participant characteristics were compared across randomization arms at baseline using $\chi^{2}$ test for categorical variables and $t$ test for continuous variables or Kruskal-Wallis test when warranted due to skewness. In instances in which urine biomarker levels were below the limit of assay detection, the value of the lower limit was imputed. Distributions of outcomes were evaluated for normality and $\log _{2}$ transformations were used to correct urine biomarker levels for positive skewness. To make presentation of all results consistent, $\log _{2}$ transformation of eGFR was also used when modeling it as an outcome. Changes in outcomes over time between randomization arms were evaluated using mixed-effects linear models in a repeated-measures layout with unstructured $3 \times 3$ variance-covariance matrix to account for within-subject correlation among the 3 measured time points (baseline, 12 months, and 48 months). All analyses were performed using the intention-to-treat approach. Exploratory analyses of urinary biomarkers demonstrated that model fit was best when terms adjusting for linear and quadratic urine creatinine concentration were included. Thus, all models for those outcomes (with the 
exception of ACR) included both linear and quadratic terms for logarithmically transformed (base 2) urine creatinine as time-varying covariates to account for differences in urine tonicity. Time (baseline, month 12, or month 48 follow-up) was treated as a class effect in all models. The difference in geometric least squares means of log-transformed urinary biomarkers between the intensive and standard BP arms was determined and backtransformation provided the ratio of geometric means and the related $95 \%$ confidence intervals (CIs).

For additional insights, we evaluated 1- and 4-year changes in urinary biomarker levels and their associations with quintiles of change in eGFR using linear analysis of covariance models that adjusted for concurrently measured urine creatinine. This approach allowed us to determine whether the magnitude of change in urine biomarker levels mirrored concurrent changes in eGFRs. We defined quintiles of eGFR change based on the distribution observed in the intensive BP arm and applied the resulting cut-points to the standard BP arm for consistency. Similarly, we stratified participants into quintiles of observed changes in SBP during the trial within the intensive BP arm and compared the magnitude of changes in urine biomarker levels across quintiles, again using linear analysis of covariance models.

\section{Results}

Among 978 SPRINT participants with baseline eGFRs $<60 \mathrm{~mL} / \mathrm{min} / 1.73 \mathrm{~m}^{2}$ included in this study, mean age was $72 \pm 9$ years, $60 \%$ were men, $66 \%$ were non-Hispanic whites, and $39 \%$ had a history of CVD. Median eGFR at baseline was 48 (interquartile range [IQR], 4054) $\mathrm{mL} / \mathrm{min} / 1.73 \mathrm{~m}^{2}$, median urinary ACR was 15 (IQR, 7-52) $\mathrm{mg} / \mathrm{g}$, and mean SBP and diastolic BP were $139 \pm 16$ and $75 \pm 12 \mathrm{~mm} \mathrm{Hg}$, respectively, at baseline. Mean number of antihypertensive medications at baseline was $2.0 \pm 1.0$. Five hundred nineteen participants were randomly assigned to the intensive BP arm, and 459 were randomly assigned to the standard BP arm. Baseline demographic, clinical, and laboratory characteristics are displayed by randomly assigned treatment arm in Table 1. Distributions of all measurements were similar across arms except for serum triglyceride levels, which were lower in the intensive BP arm (124 \pm 61 vs $135 \pm 92 \mathrm{mg} / \mathrm{dL} ; P=0.04)$.

Table S1 shows selected baseline factors among participants with CKD who were versus were not sampled for this study. These samples appear similar, albeit those sampled appeared slightly younger, had slightly lower Framingham risk scores, and had slightly lower eGFRs at baseline.

Baseline concentrations of each of the 8 urinary tubule biomarkers (B2M, A1M, UMOD, IL-18, KIM-1, NGAL, MCP-1, and YKL-40) were similar across the 2 arms. As expected from the previous publication from SPRINT, ${ }^{14}$ eGFR was $7 \%$ lower and ACR was $32 \%$ lower at year 1 among participants randomly assigned to intensive BP compared to the standard arm in the subset included in this analysis (Table 2; Fig 1). None of the 8 urinary tubular marker levels were higher in the intensive versus standard arm at year 1. Using an omnibus test to compare differences in urinary biomarker levels across baseline, year 1, and year 4, statistically significant differences were detected for 2 biomarkers: $\mathrm{B} 2 \mathrm{M}(P=0.03)$ and A1M $(P=0.01)$ across study years. Comparing differences at year $1, \mathrm{~B} 2 \mathrm{M}$ level was 
$29 \%$ (95\% CI, 10\%-43\%) lower, and A1M level was 24\% (95\% CI, 10\%-36\%) lower in the intensive arm relative to the standard arm. Although the omnibus $\mathrm{P}$ value did not reach statistical significance across years for urinary IL-18 level $(P=0.2)$, a similar difference was observed at year 1, in which IL-18 concentrations were 11\% (95\% CI, 2\%-19\%) lower in the intensive versus standard arm at year $1(P=0.01)$. At year 4 , differences in eGFRs and urinary ACRs across BP arms were similar to year 1 (7\% and 31\% lower in the intensive vs standard arm, respectively). However, we did not observe statistically significant differences in any urinary tubule marker level across arms at year 4.

We next evaluated change in urinary ACR and urine tubule marker levels in participants with extremes of eGFR changes at years 1 and 4 in both the intensive and standard BP arms. In the intensive arm, persons who experienced decreases in eGFRs among the highest quintile had the largest reductions in urinary ACR, B2M, A1M, and IL-18 levels at year 1.

Evaluation of heterogeneity across eGFR categories was statistically significant for ACR, A1M, and IL-18 (all $P<0.01)$ and approached statistical significance for B2M $(P=0.07$; Table 3). At year 4, changes in urine biomarker levels were not related to the magnitude of change in eGFR in either treatment arm. Changes in the other biomarker levels are reported in Table S2.

Next, we limited the sample to participants randomly assigned to the intensive arm and compared the relationship of the magnitude of achieved change in SBP during the trial with concurrent changes in urinary tubule marker levels. At year 1, participants in the intensive arm who achieved the largest SBP reductions (SBP decline $>30 \mathrm{~mm} \mathrm{Hg}$ from baseline) also experienced the greatest reductions in eGFRs (of 11\%) and urinary ACRs (of 40\%), whereas those with the smallest changes in SBPs experienced the least changes or improvements in eGFRs and urinary ACRs during follow-up $(P<0.001)$. Similarly, we observed a $30 \%$ decrease in urinary B2M and $41 \%$ decrease in urinary A1M levels among participants with the largest SBP reductions at year 1 and the least changes in those with smaller reductions in SBP (Table 4). These observations were no longer apparent at year 4. In comparison, we observed no consistent changes in urinary tubule marker levels across the range of achieved SBP reduction for any of the remaining markers at year 1 (Table S3).

A total of 62 deaths occurred in our study sample during a 4-year follow-up: 28 in the intensive BP arm and 34 in the standard BP arm. There were no significant between-group differences for the need for hemodialysis ( 5 in the intensive BP arm and 7 in the standard BP arm). Two participants were lost during follow-up in the intensive BP arm and none were lost in the standard BP arm.

\section{Discussion}

In this study, we have evaluated the effects of random assignment to the intensive SBPlowering arm of SPRINT on urinary markers of kidney tubule function, injury, and repair in participants with CKD. We found that random assignment to the intensive SBP arm was associated with a decline in eGFR by year 1 that persisted over 4 years. Concurrently, we found that concentrations of 2 kidney tubule function markers, urinary B2M and $\mathrm{A} 1 \mathrm{M}$, were lower in the intensive arm at 1 year, an effect that was attenuated and no longer evident by 4 
years after randomization. As we hypothesized, none of the kidney tubule cell biomarkers had a statistically higher concentration in the intensive arm at either the year 1 or year 4 follow-up visits despite the loss of eGFR in the intensive arm.

SPRINT and several other trials evaluating more versus less intensive BP lowering have demonstrated that intensive BP lowering results in acute losses of eGFR. ${ }^{8,11,12,14}$ These eGFR differences appear to persist during follow-up, but with relatively similar slopes across treatment arms after the acute phase. ${ }^{11,14}$ Determining whether intensive BP lowering reflects hemodynamic changes versus intrinsic kidney damage is of high importance given proven benefits in CVD and mortality risk reduction, but perceived potential harm on the kidney with intensive BP lowering. Here, we evaluated 8 urinary markers of intrinsic kidney tubule damage. Despite declines in eGFR in the intensive arm, we found no evidence that levels of any of the 8 kidney tubule biomarkers were elevated compared to the standard SBP arm, after either 1 or 4 years of intensive BP lowering. Because higher urine levels of these kidney tubule markers have been linked to CKD progression, dialysis therapy initiation, and adverse health outcomes, ${ }^{16,17,24-27}$ the present results provide reassurance that the eGFR decline with intensive BP lowering is likely predominantly hemodynamic in nature.

Levels of 2 of the biomarkers (urinary B2M and A1M) were significantly lower, rather than higher, in the intensive BP arm at year 1. These biomarkers of proximal tubule function share similar properties in their renal handling and therefore give insights to the biology responsible for changes in eGFR with intensive BP lowering. Both B2M and A1M are serum proteins that are filtered by the glomerulus and then reabsorbed by the proximal tubule. In contrast, the other 6 urinary tubule biomarkers are produced in kidney tissue in response to damage, inflammation, and repair and are not known to be filtered at the glomerulus. Our findings suggest that intensive SBP lowering results in a hemodynamic decrease in GFR, which not only lowers creatinine filtration, but also lowers B2M and A1M filtration in the presence of preserved tubular reabsorptive capacities, resulting in lower urine concentrations. These findings are reinforced by our analyses stratified by the magnitude of change in eGFR and SBP. Participants in the intensive arm who experienced the largest reductions in eGFR and SBP during the trial also experienced the greatest reductions in urinary B2M and A1M levels. Similarly, the finding of lower albuminuria in the intensive SBP arm may be as a consequence of decrease in glomerular capillary pressure or inhibition of podocyte damage and myofibroblast transformation and requires further investigation. ${ }^{14}$

We believe that our findings may have clinical implications because they may provide reassurance to clinicians and patients when they consider continuation of intensive BPlowering therapy even if eGFR increases within the range observed within SPRINT. We believe that chronic hemodynamic perturbations would not lead to tubular damage. For example, recent studies evaluating sodium/glucose co-transporter 2 (SGLT2) inhibitors show acute hemodynamic effects on eGFR that persist for years, but then rapidly resolve after drug discontinuation. ${ }^{28-30}$ These drugs are associated with lower risk for end-stage kidney disease. ${ }^{29}$ These findings support, but do not prove, the hypothesis that hemodynamic effects on eGFR may persist for years without necessarily causing tubule damage. In addition, our findings suggest that the tubule health markers may have utility to assess intrinsic versus hemodynamic changes in kidney function in other settings that are known to influence renal 
perfusion. Examples may include monitoring of patients initiated on treatment with angiotensin-converting enzyme inhibitors, nonsteroidal anti-inflammatory medications, SGLT2 inhibitors, and other drugs. These important questions require future study.

This study has several strengths. First, we longitudinally assessed multiple urinary kidney tubule markers that reflect unique aspects of kidney tubule biology, including tubule function, injury, inflammation, and repair. Kidney tubule cell damage, atrophy, and tubulointerstitial fibrosis are hallmarks of nearly all forms of progressive CKD, and the urinary biomarkers evaluated here are known to be associated with CKD progression above and beyond eGFR and urinary ACR. The randomized trial design, 4 years of follow-up, and consistent directions of the observed associations across the panel of biomarkers are additional strengths. The kidney tubule marker measurements were performed en bloc to minimize the influence of laboratory drift and more closely reflect biological changes. Biomarkers were measured twice in each sample and results were averaged to improve precision. The randomized trial design minimizes the influence of bias or unmeasured confounding on our results.

The study also has important limitations. First, because SPRINT excluded persons with diabetes, stroke, or proteinuria with protein excretion $>1 \mathrm{~g} / \mathrm{d}$, and few participants had advanced CKD, future research will be needed to determine whether these results are generalizable to other settings. However, similar findings in a subcohort of the ACCORD BP (Action to Control Cardiovascular Risk in Diabetes-Blood Pressure) trial ${ }^{31}$ suggest that our observations are not limited to this SPRINT subgroup. Second, we chose 8 urinary biomarkers to reflect different aspects of kidney tubule function and damage. Therefore, we cannot address whether intensive BP lowering may damage other nontubular components of the kidney, which might not be captured by the 8 biomarkers.

In conclusion, although intensive SBP lowering resulted in reductions in eGFR, we found no evidence that it induced kidney tubule cell damage based on evaluation of 8 distinct kidney tubule biomarkers. Intensive BP lowering was associated with lower concentrations of 2 urinary biomarkers that are filtered at the glomerulus and reabsorbed at the proximal tubule. These findings support the hypothesis that reductions in eGFR observed with intensive BP lowering reflect hemodynamic changes rather than intrinsic kidney cell damage in persons with CKD.

\section{Supplementary Material}

Refer to Web version on PubMed Central for supplementary material.

\section{Acknowledgments}

Support: This work was supported by the National Institutes of Health (NIH) and the National Research Service Award through National Institute of Diabetes and Digestive and Kidney Diseases (NIDDK) grants R01DK098234 and K24DK110427 (to Dr Ix), and the American Heart Association (grant 14EIA18560026 to Dr Ix). SPRINT is funded with federal funds from the NIH, including the National Heart, Lung, and Blood Institute, the NIDDK, the National Institute on Aging, and the National Institute of Neurological Disorders and Stroke, under contract numbers HHSN268200900040C, HHSN268200900046C, HHSN268 200900047C, HHSN268200900048C, HHSN268200900049C, and Inter-Agency Agreement Number A-HL-13-002-001. It was also supported in part with resources and use of facilities through the Department of Veterans Affairs. The SPRINT investigators 
acknowledge the contribution of study medications (azilsartan and azilsartan combined with chlorthalidone) from Takeda Pharmaceuticals Int, Inc. All components of the SPRINT protocol were designed and implemented by the investigators, and the investigative team collected, analyzed, and interpreted the data. We also acknowledge support from the following Clinical and Translational Science Awards funded by National Center for Advancing Translational Sciences of the NIH (Case Western Reserve University: UL1TR000439; Ohio State University: UL1RR025755; University of Pennsylvania: UL1RR024134 and UL1TR000003; Boston University: UL1RR025771; Stanford University: UL1TR000093; Tufts University: UL1RR025752, UL1TR000073 and UL1TR001064; University of Illinois: UL1TR000050; University of Pittsburgh: UL1TR000005; University of Texas Southwestern: 9U54TR000017-06; University of Utah: UL1TR000105-05; Vanderbilt University: UL1TR000445; George Washington University: UL1TR000075; University of California, Davis: UL1TR000002; University of Florida: UL1TR000064; University of Michigan: UL1TR000433; and Tulane University: P30GM103337 COBRE Award NIGMS. The funders did not have a role in study design; collection, analysis, and interpretation of the data; and writing of the manuscript.

Financial Disclosure: Dr Cheung is a consultant for Boehringer Ingelheim and a contributor to UpToDate. Dr Sarnak serves on the Steering Committee for a trial funded by Akebia. Dr Parikh is a member of the advisory board of RenalytixAI and own equity in the same. Dr Shlipak has worked as a consultant for the University of Washington and holds equity in TAI Diagnostics and Cricket Health, Inc. Dr Ix holds an investigator-initiated research grant from Baxter International for an unrelated project. The remaining authors declare that they have no relevant financial interests.

\section{References}

1. Lewington S, Clarke R, Qizilbash N, et al. Age-specific relevance of usual blood pressure to vascular mortality: a meta-analysis of individual data for one million adults in 61 prospective studies. Lancet. 2002;360(9349):1903-1913. [PubMed: 12493255]

2. Vasan RS, Larson MG, Leip EP, et al. Impact of high-normal blood pressure on the risk of cardiovascular disease. N Engl J Med. 2001;345(18):1291-129. [PubMed: 11794147]

3. Zanchetti A Hypertension-related mortality and morbidity. J Hypertens. 2015;33(10):1979-1980. [PubMed: 26431184]

4. James PA, Oparil S, Carter BL, et al. 2014 Evidence-based guideline for the management of high blood pressure in adults: report from the panel members appointed to the Eighth Joint National Committee (JNC 8). JAMA. 2014;311(5):507-520. [PubMed: 24352797]

5. Neal B, MacMahon S, Chapman N, et al. Effects of ACE inhibitors, calcium antagonists, and other blood-pressure-lowering drugs: results of prospectively designed overviews of randomised trials. Blood Pressure Lowering Treatment Trialists' Collaboration. Lancet. 2000;356(9246):1955-1964. [PubMed: 11130523]

6. Malhotra R, Nguyen HA, Benavente O, et al. Association between more intensive vs less intensive blood pressure lowering and risk of mortality in chronic kidney disease stages 3 to 5: a systematic review and meta-analysis. JAMA Intern Med. 2017;177(10):1498-1505. [PubMed: 28873137]

7. Hirsch S, Hirsch J, Bhatt U, et al. Tolerating increases in the serum creatinine following aggressive treatment of chronic kidney disease, hypertension and proteinuria: pre-renal success. Am J Nephrol. 2012;36(5):430-43. [PubMed: 23128144]

8. Klahr S, Levey AS, Beck GJ, et al. The effects of dietary protein restriction and blood-pressure control on the progression of chronic renal disease. Modification of Diet in Renal Disease Study Group. N Engl J Med. 1994;330(13):877-884. [PubMed: 8114857]

9. Lv J, Ehteshami P, Sarnak MJ, et al. Effects of intensive blood pressure lowering on the progression of chronic kidney disease: a systematic review and meta-analysis. CMAJ. 2013;185(11):949-95. [PubMed: 23798459]

10. Wright JT, Bakris G, Greene T, et al. Effect of blood pressure lowering and antihypertensive drug class on progression of hypertensive kidney disease: results from the AASK trial. JAMA. 2002;288(19):2421-2431. [PubMed: 12435255]

11. Peralta CA, McClure LA, Scherzer R, et al. Effect of intensive versus usual blood pressure control on kidney function among individuals with prior lacunar stroke: a post hoc analysis of the Secondary Prevention of Small Subcortical Strokes (SPS3) Randomized Trial. Circulation. 2016;133(6):584-591. [PubMed: 26762524]

12. Schrier RW, Abebe KZ, Perrone RD, et al. Blood pressure in early autosomal dominant polycystic kidney disease. N Engl J Med. 2014;371(24):2255-2266. [PubMed: 25399733] 
13. SPRINT Research Group; Wright JT Jr, Williamson JD, Whelton PK, et al. A randomized trial of intensive versus standard blood-pressure control. N Engl J Med. 2015;373(22): 2103-2116. [PubMed: 26551272]

14. Cheung AK, Rahman M, Reboussin DM, et al. Effects of intensive BP control in CKD. J Am Soc Nephrol. 2017;28(9): 2812-2823. [PubMed: 28642330]

15. Malhotra R, Siew ED. Biomarkers for the early detection and prognosis of acute kidney injury. Clin J Am Soc Nephrol. 2017;12(1):149-173. [PubMed: 27827308]

16. Peralta CA, Katz R, Bonventre JV, et al. Associations of urinary levels of kidney injury molecule 1 (KIM-1) and neutrophil gelatinase-associated lipocalin (NGAL) with kidney function decline in the Multi-Ethnic Study of Atherosclerosis (MESA). Am J Kidney Dis. 2012;60(6):904-911. [PubMed: 22749388]

17. Scherzer R, Lin H, Abraham A, et al. Use of urine biomarker-derived clusters to predict the risk of chronic kidney disease and all-cause mortality in HIV-infected women. Nephrol Dial Transplant. 2016;31(9):1478-1485. [PubMed: 26754833]

18. Ambrosius WT, Sink KM, Foy CG, et al. The design and rationale of a multicenter clinical trial comparing two strategies for control of systolic blood pressure: the Systolic Blood Pressure Intervention Trial (SPRINT). Clin Trials 2014;11(5):532-546. [PubMed: 24902920]

19. Jotwani V, Scherzer R, Abraham A, et al. Association of urine alpha1-microglobulin with kidney function decline and mortality in HIV-infected women. Clin J Am Soc Nephrol. 2015;10(1): 6373. [PubMed: 25370597]

20. Parikh CR, Jani A, Mishra J, et al. Urine NGAL and IL-18 are predictive biomarkers for delayed graft function following kidney transplantation. Am J Transplant. 2006;6(7): 1639-1645. [PubMed: 16827865]

21. Waikar SS, Sabbisetti V, Ärnlöv J, et al. Relationship of proximal tubular injury to chronic kidney disease as assessed by urinary kidney injury molecule-1 in five cohort studies. Nephrol Dial Transplant. 2016;31(9):1460-1470. [PubMed: 27270293]

22. Park M, Katz R, Shlipak MG, et al. Urinary markers of fibrosis and risk of cardiovascular events and death in kidney transplant recipients: the FAVORIT Trial. Am J Transplant. 2017;17(10): 2640-2649. [PubMed: 28371433]

23. Schmidt IM, Hall IE, Kale S, et al. Chitinase-like protein Brp-39/YKL-40 modulates the renal response to ischemic injury and predicts delayed allograft function. J Am Soc Nephrol. 2013;24(2):309-319. [PubMed: 23291472]

24. Ix JH, Katz R, Bansal N, et al. Urine fibrosis markers and risk of allograft failure in kidney transplant recipients: a case-cohort ancillary study of the FAVORIT Trial. Am J Kidney Dis. 2017;69(3):410-419. [PubMed: 28024930]

25. Nadkarni GN, Rao V, Ismail-Beigi F, et al. Association of urinary biomarkers of inflammation, injury, and fibrosis with renal function decline: the ACCORD Trial. Clin J Am Soc Nephrol. 2016;11(8):1343-1352. [PubMed: 27189318]

26. Hall IE, Yarlagadda SG, Coca SG, et al. IL-18 and urinary NGAL predict dialysis and graft recovery after kidney transplantation. J Am Soc Nephrol. 2010;21(1):189-19. [PubMed: 19762491]

27. Puthumana J, Hall IE, Reese PP, et al. YKL-40 associates with renal recovery in deceased donor kidney transplantation. J Am Soc Nephrol. 2017;28(2):661-670. [PubMed: 27451287]

28. Cherney DZ, Perkins BA, Soleymanlou N, et al. Renal hemodynamic effect of sodium-glucose cotransporter 2 inhibition in patients with type 1 diabetes mellitus. Circulation. 2014;129(5): 58759. [PubMed: 24334175]

29. Tang H, Li D, Zhang J, et al. Sodium-glucose cotransporter 2 inhibitors and risk of adverse renal outcomes among type 2 diabetes patients: a network and cumulative meta-analysis of randomized controlled trials. Diabetes Obes Metab. 2017;19(8):1106-1115. [PubMed: 28240446]

30. Wanner C, Inzucchi SE, Lachin JM, et al. Empagliflozin and progression of kidney disease in type 2 diabetes. N Engl J Med. 2016;375(18):323-334. [PubMed: 27299675]

31. Nadkarni GN, Chauhan K, Rao V, et al. Effect of intensive blood pressure lowering on kidney tubule injury: findings from the ACCORD Trial study participants. Am J Kidney Dis. 2018;73(1):31-38. [PubMed: 30291011] 


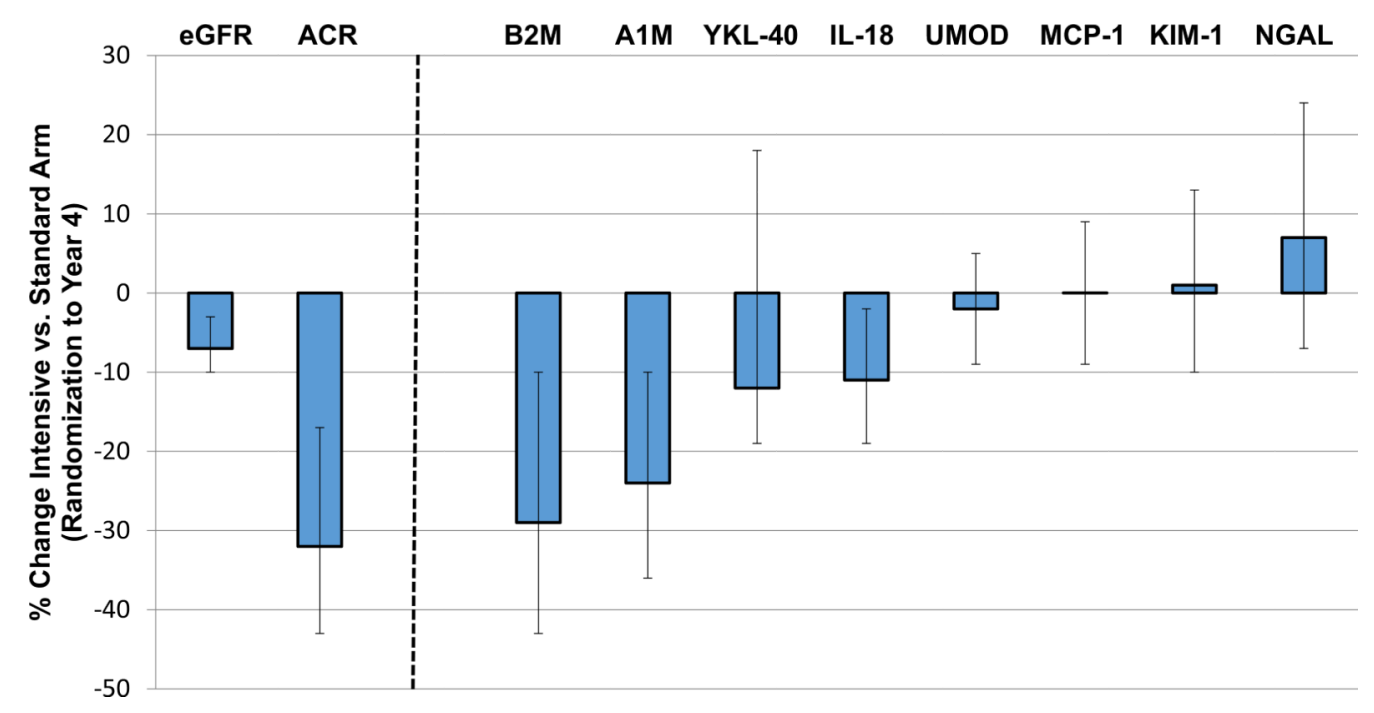

Figure 1.

Percentage 1-year change between intensive versus standard blood pressure control for estimated glomerular filtration rate, albuminuria, and urinary tubular markers in participants with chronic kidney disease in SPRINT (Systolic Blood Pressure Intervention Trial). Abbreviations: A1M, $a_{1}$-microglobulin; ACR, albumin-creatinine ratio; B2M, $\beta_{2}$ microglobulin; eGFR, estimated glomerular filtration rate; KIM-1, kidney injury molecule 1; IL-18, interleukin 18; MCP-1, monocyte chemoattractant protein 1; NGAL, neutrophil gelatinase-associated lipocalin; UMOD, uromodulin; YKL-40, human cartilage glycoprotein 39. 
Table 1.

Baseline Characteristics of Study Participants by Randomized Treatment Arm

\begin{tabular}{llll}
\hline & Intensive BP Arm $(\mathbf{n}=\mathbf{5 1 9})$ & Standard BP Arm $(\mathbf{n}=\mathbf{4 5 9})$ & $\boldsymbol{P}$ \\
\hline Age, $\mathrm{y}$ & $72 \pm 9$ & $72 \pm 9$ & 0.9 \\
\hline Male sex & $304(59 \%)$ & $280(61 \%)$ & 0.4 \\
\hline White & $345(67 \%)$ & $302(66 \%)$ & 0.8 \\
\hline Education: some college or greater & $376(73 \%)$ & $318(69 \%)$ & 0.3 \\
\hline Current smoker & $232(45 \%)$ & $205(45 \%)$ & 0.9 \\
\hline History of heart disease & $212(41 \%)$ & $166(36 \%)$ & 0.1 \\
\hline History of PVD & $42(8 \%)$ & $44(10 \%)$ & 0.4 \\
\hline SBP, $\mathrm{mm}$ Hg & $139 \pm 16$ & $140 \pm 17$ & 0.4 \\
\hline DBP, $\mathrm{mm} \mathrm{Hg}$ & $75 \pm 12$ & $74 \pm 12$ & 0.9 \\
\hline No. of BP medications & $2 \pm 1$ & $2 \pm 1$ & 0.5 \\
\hline Treated by diuretic & $199(38 \%)$ & $269(59 \%)$ & 0.3 \\
\hline Treated by ARB or ACEi & $253(49 \%)$ & $229(50 \%)$ & 0.7 \\
\hline Total cholesterol, $\mathrm{mg} / \mathrm{dL}$ & $185 \pm 41$ & $182 \pm 39$ & 0.3 \\
\hline HDL cholesterol, $\mathrm{mg} / \mathrm{dL}$ & $52 \pm 14$ & $52 \pm 15$ & 0.9 \\
\hline Triglycerides, $\mathrm{mg} / \mathrm{dL}$ & $124 \pm 61$ & $135 \pm 92$ & 0.04 \\
\hline Body mass index, $\mathrm{kg} / \mathrm{m}^{2}$ & $30 \pm 6$ & $29 \pm 6$ & 0.09 \\
\hline eGFR, $\mathrm{mL} / \mathrm{min} / 1.73 \mathrm{~m}{ }^{2}$ & $48[39-54]$ & $48[41-54]$ & 0.7 \\
\hline Urinary ACR, $\mathrm{mg} / \mathrm{g}$ & $15[7-51]$ & $16[7-56]$ & 0.9 \\
\hline
\end{tabular}

Note: Values for continuous variables are given as mean \pm standard deviation or median [interquartile range]; those for categorical variables, as count (percentage).

Abbreviations: ACEi, angiotensin-converting enzyme inhibitor; ACR, albumin-creatinine ratio; ARB, angiotensin II receptor blocker; BP, blood pressure; DBP, diastolic blood pressure; eGFR, estimated glomerular filtration rate; HDL, high-density lipoprotein; PVD, peripheral vascular disease; SBP, systolic blood pressure.

${ }^{a} P$ value from Kruskal-Wallis test for difference in median values. 


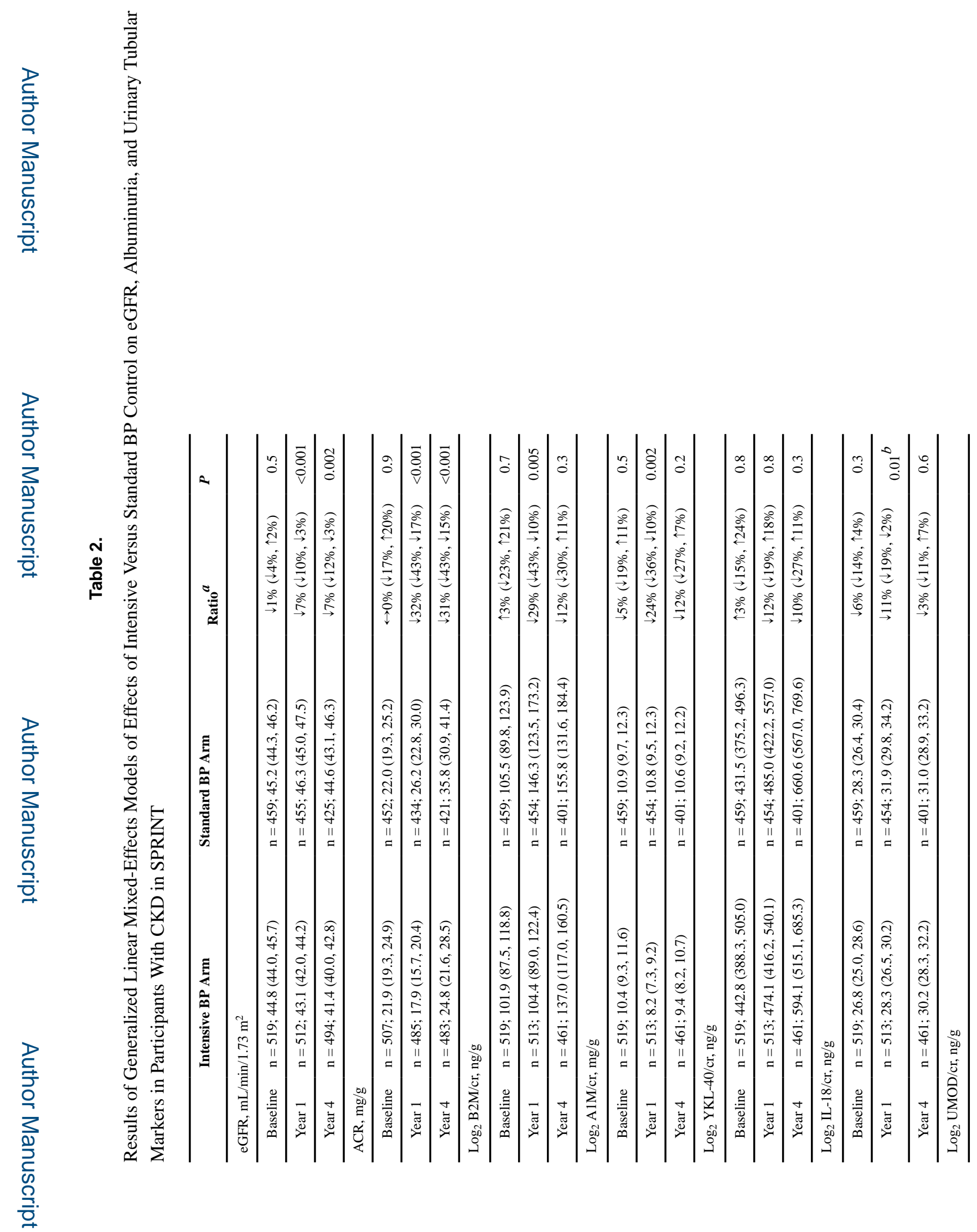

Am J Kidney Dis. Author manuscript; available in PMC 2020 June 30. 


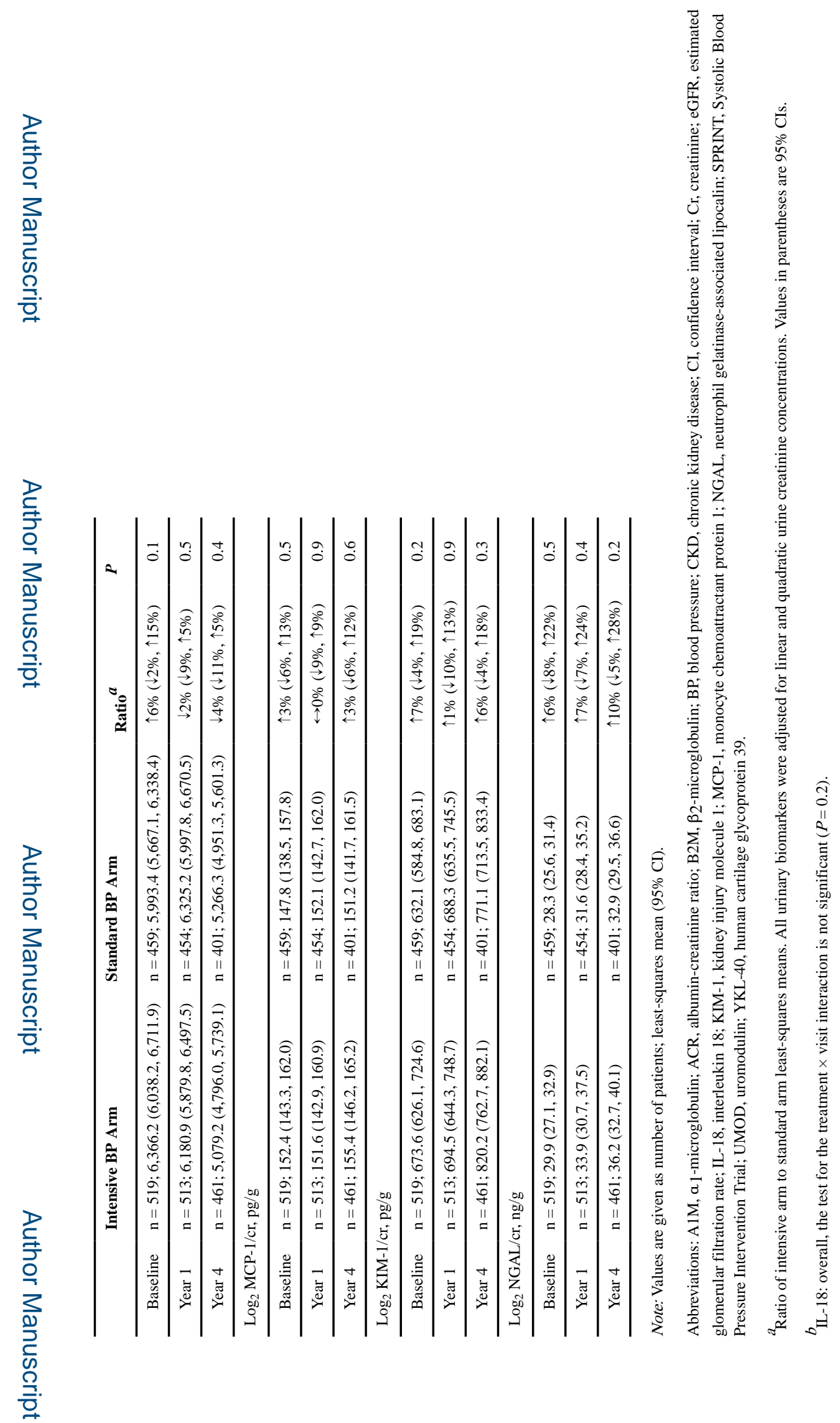




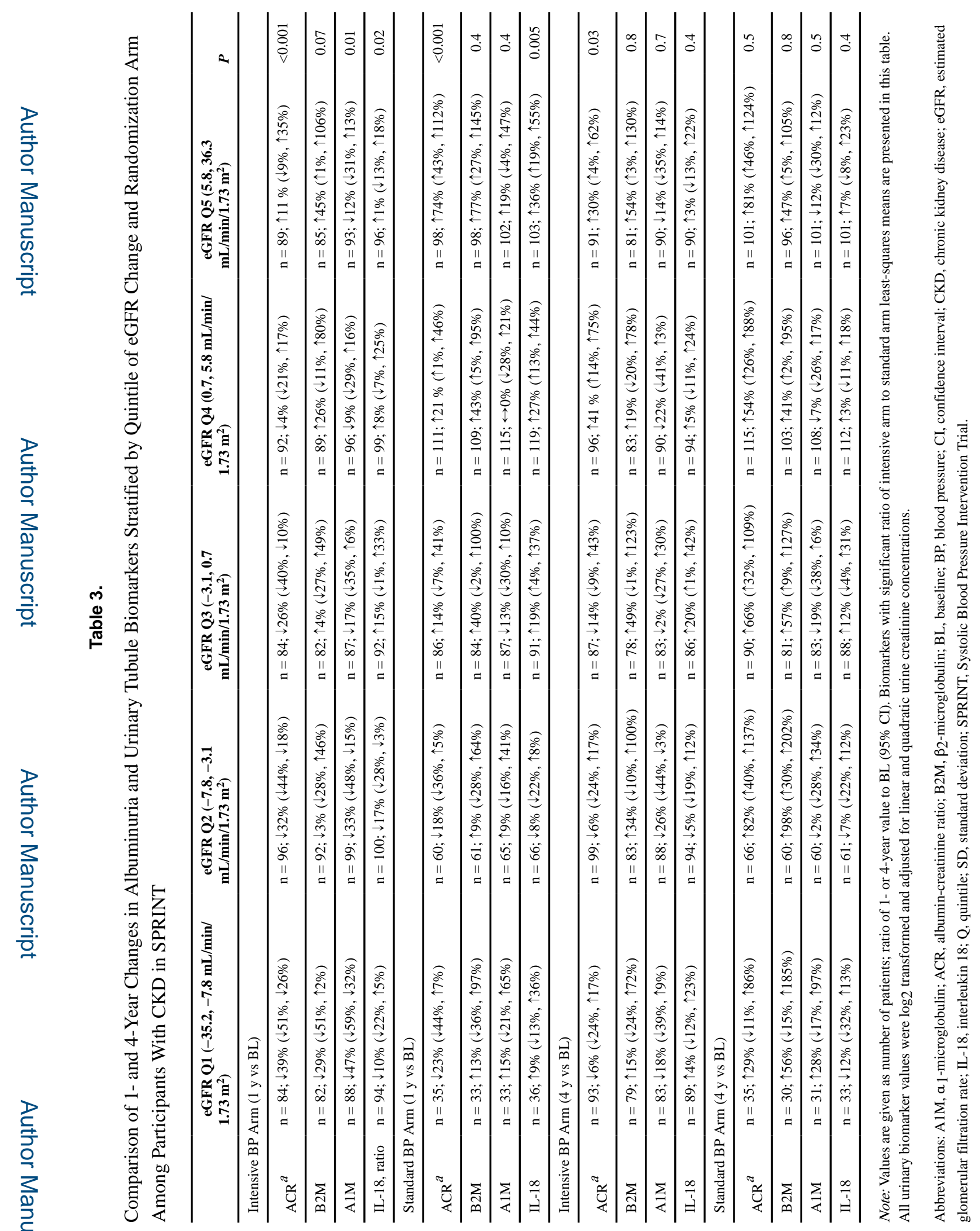

Am J Kidney Dis. Author manuscript; available in PMC 2020 June 30. 


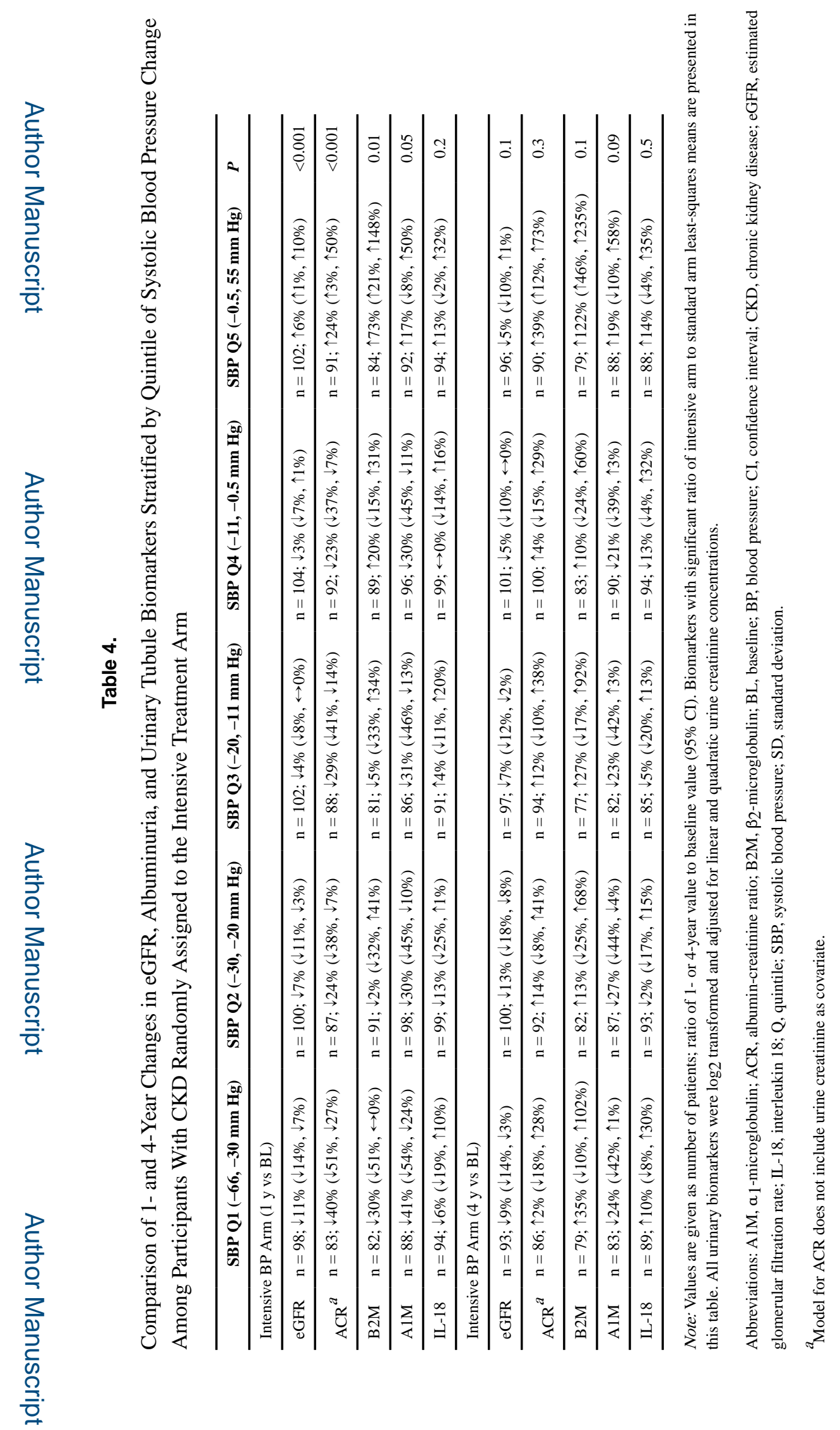

Am J Kidney Dis. Author manuscript; available in PMC 2020 June 30. 